\title{
Noncrossing sets and a Graßmann associahedron
}

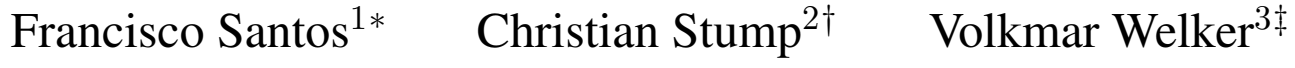 \\ ${ }^{1}$ Departamento de Matemáticas, Estadística y Computación, Universidad de Cantabria, Santander, Spain \\ ${ }^{2}$ Institut für Mathematik, Freie Universität Berlin, Germany \\ ${ }^{3}$ Fachbereich Mathematik und Informatik, Philipps-Universität Marburg, Germany
}

\begin{abstract}
We study a natural generalization of the noncrossing relation between pairs of elements in $[n]$ to $k$-tuples in $[n]$. We show that the flag simplicial complex on $\left(\begin{array}{c}{[n]} \\ k\end{array}\right)$ induced by this relation is a regular, unimodular and flag triangulation of the order polytope of the poset given by the product $[k] \times[n-k]$ of two chains, and it is the join of a simplex and a sphere (that is, it is a Gorenstein triangulation). This shows the existence of a flag simplicial polytope whose Stanley-Reisner ideal is an initial ideal of the Graßmann-Plücker ideal, while previous constructions of such a polytope did not guaranteed flagness. The simplicial complex and the polytope derived from it naturally reflect the relations between Graßmannians with different parameters, in particular the isomorphism $G_{k, n} \cong G_{n-k, n}$. This simplicial complex is closely related to the weak separability complex introduced by Zelevinsky and Leclerc.
\end{abstract}

Résumé: Nous étudions une généralisation naturelle de la relation entre les paires d'éléments non-croisés de $[n]$ et les $k$-uplets de $[n]$. Nous montrons que le complexe simplicial de drapeau sur $\left(\begin{array}{c}{[n]} \\ k\end{array}\right)$ induit par cette relation est une triangulation régulière, unimodulaire et de drapeau du polytope d'ordre de l'ensemble partiellement ordonné obtenu par le produit $[k] \times[n-k]$ des deux chaînes, et c'est la jointure d'un simplexe et une sphère (c'est-à-dire qu'elle est une triangulation de Gorenstein). Cela montre l'existence d'un polytope simplicial de drapeau dont l'idéal de Stanley-Reisner est un idéal initial de l'idéal de Graßmann-Plücker, tandis que les constructions précédentes d'un tel polytope ne garantissaient pas la propriété de drapeau. Le complexe simplicial et le polytope qui en découle reflètent naturellement les relations entre les Grassmanniens avec différents paramètres, en particulier l'isomorphisme $G_{k, n} \cong G_{n-k, n}$. Ce complexe simplicial est étroitement lié au complexe de séparabilité faible étudié par Zelevinsky et Leclerc.

Keywords: Graßmannian, associahedron, crossing, order polytope, triangulation

\footnotetext{
*Email: francisco.santos@unican.es. Supported by the Spanish Ministry of Science (MICINN) through grant MTM2011-22792, and by a Humboldt Research Award of the Alexander von Humboldt Foundation.

†Email: christian.stump@fu-berlin. de. Supported by the German Research Foundation DFG, grant STU 563/2-1 "Coxeter-Catalan combinatorics".

‡Email: welker@mathematik.uni-marburg.de.
}

1365-8050 (c) 2014 Discrete Mathematics and Theoretical Computer Science (DMTCS), Nancy, France 


\section{Introduction}

Let $[n]$ denote the set $\{1, \ldots, n\}$ of the first $n$ positive integers. Two pairs $\left(i<i^{\prime}\right)$ and $\left(j<j^{\prime}\right)$ with $i \leq j$ are said to nest if $i<j<j^{\prime}<i^{\prime}$ and cross if $i<j<i^{\prime}<j^{\prime}$. In other words, they nest and cross if the two arcs nest and, respectively, cross in the following picture,

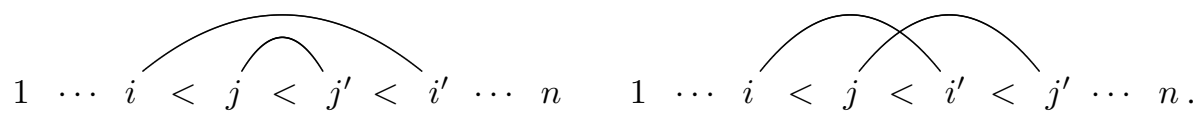

Nestings and crossings have been intensively studied and generalized in the literature, see e.g. [1, 12, 14]. One important context in which they appear are two pure and flag simplicial complexes $\Delta_{n}^{N N}$ and $\Delta_{n}^{N C}$. Recall here that a flag simplicial complex is the complex of cliques of a graph. The complex $\Delta_{n}^{N N}$ is the flag complex having the arcs $1 \leq i<j \leq n$ as vertices and pairs of nonnesting arcs as edges, while the complex $\Delta_{n}^{N C}$ is the flag complex with the same vertices and pairs of noncrossing arcs as edges.

It is not hard to see that the facets of $\Delta_{n}^{N N}$ are given by Dyck paths of length $2(n-2)$, while the facets of $\Delta_{n}^{N C}$ are given by triangulations of a convex $n$-gon. Thus in either case the facets are counted by the $(n-2)^{\text {nd }}$ Catalan number $\frac{1}{n-1}\left(\begin{array}{c}2 n-4 \\ n-2\end{array}\right)$. Moreover, it can be shown that their face vectors coincide and that both are balls of dimension $2 n-4$. In addition, the complex $\Delta_{n}^{N C}$ is the join of an $(n-1)$-simplex and an ubiquitous $(n-4)$-dimensional polytopal sphere $\widetilde{\Delta}_{n}^{N C}$, the (dual of the) associahedron.

\subsection{The nonnesting complex}

The following generalization of the nonnesting complex is well known. Let $V_{k, n}$ denote the set of all vectors $\left(i_{1}, \ldots, i_{k}\right), 1 \leq i_{1}<\cdots<i_{k} \leq n$, of length $k$ with entries in $[n]$.

Definition 1.1 Two vectors $I=\left(i_{1}, \ldots, i_{k}\right)$ and $J=\left(j_{1}, \ldots, j_{k}\right)$ in $V_{k, n}$ are nonnesting iffor all indices $a<b$ the $\operatorname{arcs}\left(i_{a}<i_{b}\right)$ and $\left(j_{a}<j_{b}\right)$ are nonnesting. The (multidimensional) nonnesting complex $\Delta_{k, n}^{N N}$ is the flag simplicial complex with vertices $V_{k, n}$ and with edges being the nonnesting pairs of vertices.

By definition, we have $\Delta_{2, n}^{N N}=\Delta_{n}^{N N}$. The non nesting complex $\Delta_{k, n}^{N N}$ can be understood as a triangulation of the order polytope of the product of a $k$-chain with an $(n-k)$-chain, as we now review. This implies that it is a simplicial ball of dimension $k(n-k)$. Moreover, through this connection its $h$-vector is linked to the Hilbert series of the coordinate ring of the Graßmannian $G_{k, n}$ of $k$-planes in $\mathbb{C}^{n}$. See details on this in Section 1.3

Recall that the order polytope $\mathcal{O}(P)$ of a poset $P$ is the polytope in $\mathbb{R}^{P}$ with vertices being the characteristic vectors of upclosed subsets or filters in $P, \mathcal{O}(P)=\operatorname{conv}\left\{\chi_{I} \quad: \quad I\right.$ filter in $\left.P\right\}$, see [18]. It is well known that the flag complex with edges given by the pairs of comparable filters in $P$ is a regular, unimodular and flag triangulation of $\mathcal{O}(P)$, that we will call the standard triangulation of $\mathcal{O}(P)$. Facets of this triangulation are in natural bijection to the linear extensions of $P$. Let now $P_{k, n}=[k] \times[n-k]$ be the product poset of chains of lengths $k$ and $n-k$, and abbreviate its order polytope by $\mathcal{O}_{k, n}$. It is a simple exercise to show that filters in $P_{k, n}$ are in one to one correspondence with vectors in $V_{k, n}$ via a bijection which maps pairs of comparable filters to pairs of nonnesting vectors. Using this identification, the simplicial complex $\Delta_{k, n}^{N N}$ equals the standard triangulation of $\mathcal{O}_{k, n} \subset[0,1]^{P_{k, n}}$. For two order filters $I$ and $J$ in a poset $P$, denote by $\mathcal{F}(I, J)$ the minimal face of $\mathcal{O}(P)$ containing the corresponding vertices $\chi_{I}$ and $\chi_{J}$. It turns out that $\mathcal{F}(I, J)$ is always (affinely equivalent to) a cube. Although this is not difficult to prove, this auxiliary result was new to us and relates the nonnesting and the noncrossing complexes 
(which we will define in Section 1.2) to two well known triangulations of the cube, see Remark 3.7. In the next statement we use the notation $\vec{X}$ for the segment going from the origin to an $X \in \mathbb{R}^{P}$.

Lemma 1.2 Let $I \subset J$ be two comparable filters in a poset $P$ and let $P_{1}^{\prime}, \ldots, P_{d}^{\prime}$ be the connected components of $\left.P\right|_{J \backslash I}$. Then the minimal face $\mathcal{F}(I, J)$ of the order polytope $\mathcal{O}(P)$ containing the vertices $\chi_{I}$ and $\chi_{J}$ is the Minkowski sum $\chi_{I}+\vec{\chi}_{P_{1}^{\prime}}+\cdots+\vec{\chi}_{P_{d}^{\prime}}$. Combinatorially $\mathcal{F}(I, J)$ is a cube of dimension $d$.

Corollary 1.3 For any two filters $I$ and $J$ of the poset $P$ we have $\mathcal{F}(I, J)=\mathcal{F}(I \cap J, I \cup J)$. In particular, $\mathcal{F}(I, J)$ is a cube of dimension equal to the number of connected components of $\left.P\right|_{I \triangle J}$, where $I \triangle J=(I \cup J) \backslash(I \cap J)$ denotes the symmetric difference.

Linear extensions of $P_{k, n}$, that is, facets of $\Delta_{k, n}^{N N}$, are in bijection with standard tableaux of shape $k \times(n-k)$. Here, a tableau of shape $k \times(n-k)$ is a matrix in $\mathbb{N}^{k \times(n-k)}$ that is weakly increasing along rows from left to right and along columns from bottom to top. Equivalently, it is a weakly order preserving map $P_{k, n} \rightarrow \mathbb{N}$. A tableau is called standard if it contains every integer 1 through $k(n-k)$ exactly once. An application of the hook length formula implies that facets of $\Delta_{k, n}^{N N}$ are counted by the $(n-k, k)^{\text {th }}$ multidimensional Catalan number Cat $_{n-k, k}:=\frac{0 ! 1 ! \cdots(k-1) !}{(n-1) !(n-2) ! \cdots(n-k) !}(k(n-k)) !$. These numbers were studied e.g. in [6, 20], see as well [17, Seq. A060854]. Denote the $h$-vector of $\Delta_{k, n}^{N N}$ by $\left(h_{0}^{(k, n)}, \ldots, h_{n(n-k)}^{(k, n)}\right)$. It follows from the connection of $\Delta_{k, n}^{N N}$ to the Hilbert series of the Graßmannian and was also observed in [20], going back to P. A. MacMahon's study of plane partitions, that its entries are the multidimensional Narayana numbers. We refer to [20] for an explicit formula of these numbers, which can be combinatorially defined in terms of standard tableaux of shape $k \times(n-k)$ as follows. Call peaks of a standard tableau $T$ the numbers $a \in[k(n-k)-1]$ for which $a+1$ is placed in a lower row than $a$. Then, $h_{i}^{(k, n)}$ equals the number of standard tableaux with exactly $i$ peaks. This combinatorial interpretation implies in particular that

$$
h_{i}^{(k, n)}=\left\{\begin{array}{ll}
1 & \text { if } i=k(n-k)-n+1 \\
0 & \text { if } i>k(n-k)-n+1
\end{array} .\right.
$$

\subsection{The noncrossing complex}

The reformulation of the nonnesting complex as the standard triangulation of $\mathcal{O}_{k, n}$ raises the question whether an analogous construction of a multidimensional noncrossing complex has interesting properties as well. The main result of this paper is that the following slight modification of Definition 1.1 gives a pure simplicial complex with properties as nice, and in some respects nicer, than the nonnesting complex (i)

Definition 1.4 Two vectors $I=\left(i_{1}, \ldots, i_{k}\right)$ and $J=\left(j_{1}, \ldots, j_{k}\right)$ in $V_{k, n}$ are noncrossing if for all indices $a<b$ with $i_{\ell}=j_{\ell}$ for $a<\ell<b$, the arcs $\left(i_{a}<i_{b}\right)$ and $\left(j_{a}<j_{b}\right)$ do not cross. The

(i) Note added in proof: After this paper was accepted, David Speyer has informed us that Definition 1.4 and several of our results (including Theorem 1.5 have appeared before in K. Petersen, P. Pylyavskyy, and D. Speyer, "A non-crossing standard monomial theory", Journal of Algebra, 324 (2010), 951-969. With this additional information, the main contributions of the present submission are I. the definition and first properties of the Graßmann-Tamari order briefly discussed in Remark 1.9 II. general structural results on order polytopes together with combinatorial insights that allow the parallel development of the noncrossing and nonnesting theory in Section 2 (in particular, this yields a unified deduction of the fact that the noncrossing and nonnesting complexes are flag unimodular triangulations of the order polytope $\mathcal{O}_{k, n}$ in Corollary 3.3. III. the observation that the weak separability complex equals the intersection of all cyclic shifts of the noncrossing complex (Proposition 1.10 ), IV. the discussion of the restriction of the nonnesting and noncrossing triangulations to hypercubes as discussed in Remark 3.7 
(multidimensional) noncrossing complex $\Delta_{k, n}^{N C}$ is the flag simplicial complex with vertices $V_{k, n}$ and with edges being the noncrossing pairs of vertices.

The reader may ask why in the noncrossing world we require the property only for some pairs of coordinates $a<b$, while in the nonnesting world was required for all pairs. One answer is that the direct analogue of Definition 1.1 does not even yield a pure complex. But another answer is that it would not make a difference in Definition 1.1 to require the condition only for pairs with $i_{\ell}=j_{\ell}$ for $a<\ell<b$. All pairs would automatically be nonnesting, thanks to the following transitivity of nonnestingness: let $a<b<c$ and suppose that the $\operatorname{arcs}\left(i_{a}<i_{b}\right)$ and $\left(j_{a}<j_{b}\right)$ are nonnesting, and the arcs $\left(i_{b}<i_{c}\right)$ and $\left(j_{b}<j_{c}\right)$ are nonnesting as well. Then the arcs $\left(i_{a}<i_{c}\right)$ and $\left(j_{a}<j_{c}\right)$ are also nonnesting.

The following theorem is the main result of this paper, combining Theorem 3.4 and Corollary 3.6

Theorem 1.5 The noncrossing complex $\Delta_{k, n}^{N C}$ is a flag, regular, unimodular and Gorenstein triangulation of the order polytope $\mathcal{O}_{k, n}$. In particular, $\Delta_{k, n}^{N C}$ and $\Delta_{k, n}^{N N}$ have the same $f$ - and h-vectors.

The claim that $\Delta_{k, n}^{N C}$ is "in some respects nicer" than $\Delta_{k, n}^{N N}$ is made precise in the following list of purely combinatorial properties of $\Delta_{k, n}^{N C}$ that are easy to prove directly from the definition, and which generalize well known properties of the dual associahedron to higher parameters $k$.

Proposition 1.6 The complex $\Delta_{k, n}^{N C}$ has the following properties.

(i) $I, J \in V_{k, n}$ are noncrossing if and only if they are noncrossing when restricting to $I \triangle J$.

(ii) The map $a \mapsto n+1-a$ induces an automorphism on $\Delta_{k, n}^{N C}$.

(iii) The map $I \mapsto[n] \backslash I$ induces an isomorphism $\Delta_{k, n}^{N C} \stackrel{\sim}{\longrightarrow} \Delta_{n-k, n}^{N C}$.

(iv) For $b \in[n]$ The restriction of $\Delta_{k, n}^{N C}$ to vertices with $b \in I$ yields $\Delta_{k-1, n-1}^{N C}$. The restriction of $\Delta_{k, n}^{N C}$ to vertices with $b \notin I$ yields $\Delta_{k, n-1}^{N C}$.

(v) The $n$ vertices in $V_{k, n}$ obtained by cyclic rotations of the vertex $(1,2, \ldots, k) \in V_{k, n}$ do not cross any other vertex in $V_{k, n}$ and hence are contained in every facet of $\Delta_{k, n}^{N N}$.

Properties (ii), (iii), (iii), and (iv) also hold for the nonnesting complex $\Delta_{k, n}^{N N}$, while the final Property (V) fails. Observe that Property (iii) is natural when considering the relation between $\mathcal{O}_{k, n}$ and the Graßmannian (see Section 1.3 as it reflects the fact that $G_{k, n} \cong G_{n-k, n}$. It immediately follows from Property (v) that $\Delta_{k, n}^{N C}$ is the join of an $(n-1)$-dimensional simplex and a complex $\widetilde{\Delta}_{k, n}^{N C}$ of dimension $k(n-k)-n$ such that $\widetilde{\Delta}_{k, n}^{N C}$ has the same $h$-vector as $\Delta_{k, n}^{N C}$. Put differently, $\widetilde{\Delta}_{k, n}^{N C}$ is a Gorenstein triangulation of $\mathcal{O}_{k, n}$, compare [3] and [8, Proposition 17].

Since $\widetilde{\Delta}_{2, n}^{N C}$ is the (dual) associahedron $\widetilde{\Delta}_{n}^{N C}$, and since the Stanley-Reisner ideal of $\widetilde{\Delta}_{k, n}^{N C}$ is an initial ideal for the Graßmannian, we call $\widetilde{\Delta}_{k, n}^{N C}$ the (dual) Graßmann associahedron. The Graßmann associahedron is the dual complex of $\widetilde{\Delta}_{k, n}^{N C}$. It has vertices given by the facets of $\Delta_{k, n}^{N C}$, edges given by pairs of facets that share a codimension 1 face, etc. The following statement says that the Graßmann associahedron can be realized as a simple polytope of dimension $k(n-k)-n+1=(k-1)(n-k-1)$.

Corollary 1.7 The dual Graßmann associahedron $\widetilde{\Delta}_{k, n}^{N C}$ is a flag polytopal sphere of dimension $k(n-$ $k)-n$. Moreover, Properties (iii), (iii), and (iv) in Proposition 1.6 also hold for $\widetilde{\Delta}_{k, n}^{N C}$. 
Remark 1.8 Proposition 1.6 ii] says that $\Delta_{k, n}^{N C}$ and $\widetilde{\Delta}_{k, n}^{N C}$ possess the reflection symmetry present in the associahedron $\widetilde{\Delta}_{n}^{N C}$. Of course, another symmetry of $\widetilde{\Delta}_{n}^{N C}$ comes from the cyclic rotation $i \mapsto i+1$ $(\bmod n)$. That symmetry does not carry over for $k \geq 3$, which raises the question whether there is some regular, unimodular triangulation of $\mathcal{O}_{k, n}$ implementing this cyclic symmetry. The answer seems to be no: one can show that no flag complex on the set of vertices $V_{3,6}$ that is invariant under cyclic rotation can have the h-vector $(1,10,20,10,1)$ of $\Delta_{3,6}^{N N}$.

Remark 1.9 In the long version of this extended abstract we will also present a natural way of acyclically orienting the dual graph of $\Delta_{k, n}^{N C}$. This Graßmann-Tamari poset $\mathcal{T}_{k, n}$ on maximal noncrossing subsets of $V_{k, n}$ appears to serve as an analogue of the well known Tamari order on Dyck paths. In particular, there is a linear extension of $\mathcal{T}_{k, n}$ that is a shelling order for $\Delta_{k, n}^{N C}$. This implies that the out-degree generating function of its Hasse diagram yields the multidimensional Narayana polynomial,

$$
h_{i}^{(k, n)}=\mid\left\{T \in \Delta_{k, n}^{N C}: T \text { has } i \text { upper covers in } \mathcal{T}_{k, n}\right\} \mid
$$

We moreover conjecture that the Graßmann-Tamari poset is a lattice, and raise the question whether there is an operation on peaks of standard tableaux that describes the Graßmann-Tamari order directly on standard tableaux and which generalizes the Tamari order as defined on Dyck paths.

\subsection{Motivation: the Hilbert series of the Plücker embedding}

Besides its well behaved combinatorial properties, our main motivation for studying the noncrossing complex comes from the connection between the order polytope $\mathcal{O}_{k, n}$, initial ideals of the ideal of Plücker relations, and Hilbert series of Graßmannians. We refer to [19] and [7] for more details of this connection.

Let $G_{k, n}$ denote the Graßmannian of $k$-dimensional linear subspaces in $\mathbb{C}^{n}$, and let $I_{k, n}$ be the defining ideal of the Graßmannian in its Plücker embedding. $I_{k, n}$ is the homogeneous ideal in the polynomial ring $T_{k, n}=\mathbb{C}\left[x_{i_{1}, \ldots, i_{k}}: 1 \leq i_{1}<\cdots<i_{k} \leq n\right]$ generated by the Plücker relations. It follows from Sturmfels [19] that the Stanley-Reisner ideals of regular unimodular triangulations of $\mathcal{O}_{k, n}$ are squarefree initial ideals of $I_{k, n}$. Indeed, let $J_{k, n}$ be the ideal in the polynomial ring with variables $\left\{x_{I}: I\right.$ a filter of $\left.P_{k, n}\right\}$ generated by the binomials $x_{I} x_{J}-x_{I \cap J} x_{I \cup J}$ for all choices of $I$ and $J . J_{k, n}$ is known as the Hibi ideal of the poset $P_{k, n}$, or the Ehrhart ideal of the polytope $\mathcal{O}_{k, n}$. In [19. Prop. 11.10, Cor. 8.9] it is shown that $J_{k, n}$ is an initial ideal of $I_{k, n}$. In turn, it follows from [19, Ch. 8] that there is a one to one correspondence between regular unimodular triangulations of $\mathcal{O}_{k, n}$ and squarefree monomial initial ideals of $J_{k, n}$, sending a particular regular unimodular triangulation to its Stanley-Reisner ideal.

- The regular, unimodular, flag triangulation $\Delta_{k, n}^{N N}$ of $\mathcal{O}_{k, n}$ leads to a squarefree monomial initial ideal of $J_{k, n}$ studied by T. Hibi [7].

- The regular, unimodular, flag triangulation $\Delta_{k, n}^{N C}$ provides another initial ideal with particular nice properties and leads to further insight in the Hilbert series of the coordinate ring $A_{k, n}=T_{k, n} / I_{k, n}$.

From the relation between initial ideals and unimodular triangulations stated above it follows that this Hilbert series is given by $\mathcal{H}_{A_{k, n}}(t)=H(t) /(1-t)^{k(n-k)+1}$, where $H(t)=h_{0}^{(k, n)}+h_{1}^{(k, n)} t+\cdots+$ $h_{k(n-k)}^{(k, n)} t^{k(n-k)}$ is the $h$-polynomial of any regular unimodular triangulation corresponding of $\mathcal{O}_{k, n}$, that is, the multidimensional Narayana numbers.

In the following, let $\Delta$ be a simplicial complex whose Stanley-Reisner ideal appears as an initial ideal of $I_{k, n}$. Then the following properties are desirable for $\Delta$ : 
- It follows from (1) that at most $n$ variables do not appear in the set of generators of $I_{\Delta}$. Equivalently, if $\Delta$ decomposes into $\Delta=2^{V} * \Delta^{\prime}$ where $2^{V}$ is the full simplex spanned by $V$, then $\# V \leq n$. Thus the 'most factorizable' complex $\Delta$ should be a join over a simplex spanned by $n$ vertices.

- The fact that $A_{k, n}$ is Gorenstein should be reflected in $\Delta$. Thus, $\Delta$ should be the join of a simplex with a (homology) sphere of the appropriate dimension or, even better, the boundary complex of a simplicial polytope, which would then deserve the name (dual) Graßmann associahedron.

- Since $A_{k, n}$ has a quadratic Gröbner basis, it is Koszul. Hence, one could hope that $I_{\Delta}$ is generated by quadratic monomials, or, equivalently, that $\Delta$ is flag.

- One could hope that $\Delta$ reflects the duality between $G_{k, n}$ and $G_{n-k, n}$, as well as the embeddings $G_{k-1, n-1} \hookrightarrow G_{k, n}$ and $G_{k, n-1} \hookrightarrow G_{k, n}$.

Theorem 1.5 , Proposition 1.6 and Corollary 1.7 say that $\Delta=\Delta_{k, n}^{N C}$ fulfills all these properties.

\subsection{Relation to previous work}

Weakly separable sets: Closely related to our complex is the notion of weakly separable subsets of $[n]$, introduced by B. Leclerc and A. Zelevinsky in [10] in the context of quasi-commuting families of quantum Plücker coordinates. Restricted to subsets of the same size $k$, which is the case of interest to us, the definition is that two $k$-subsets $X, Y \subset[n]$ are weakly separable if, when considered as subsets of vertices in an $n$-gon, the convex hulls of $X \backslash Y$ and $Y \backslash X$ are disjoint. The flag complex $\Delta_{k, n}^{S e p}$ of weakly separable $k$-subsets of $[n]$ was studied by J. S. Scott in [15, 16], who conjectured that $\Delta_{k, n}^{S e p}$ is pure of dimension $k(n-k)$, and that it is strongly connected (that is, its dual graph is connected). Both conjectures were shown to hold by S. Oh, A. Postnikov and D. Speyer [11] (for the first one see also V. I. Danilov, A. V. Karzanov, and G. A. Koshevoy [4, Prop. 5.9]).

It is not hard to see that $\Delta_{k, n}^{S e p}$ is a subcomplex of $\Delta_{k, n}^{N C}$ and it is trivial to observe that $\Delta_{k, n}^{S e p}$ is invariant under cyclic (or, more strongly, dihedral) symmetry. Our next result combines these two properties and

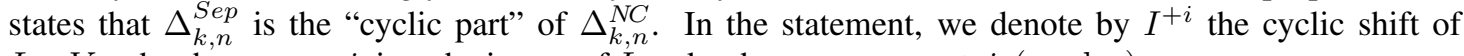
$I \in V_{k, n}$ by the amount $i$, i.e., the image of $I$ under the map $x \rightarrow x+i(\bmod n)$.

Proposition 1.10 Let $I, J \in V_{k, n}$.

1. If I and $J$ are weakly separated, then they are noncrossing.

2. If $I^{+i}$ and $J^{+i}$ are noncrossing for every $i \in[n]$, then $I$ and $J$ are weakly separated.

(The proof, based on Proposition 1.6 ii], can be found in the full version of the paper).

Put differently, the weak separation graph is the intersection of all cyclic shifts of the noncrossingness graph. Since flagness is preserved by intersection, the same happens for the complexes. We hope our approach to the noncrossing complex $\Delta_{k, n}^{N C}$ to also shed further light on the weak separability complex $\Delta_{k, n}^{S e p}$.

Triangulations of order polytopes: Triangulations of $\mathcal{O}_{k, n}$ and more generally of order polytopes $\mathcal{O}(P)$ of posets $P$ were previously known. The first unimodular and flag triangulation of order polytopes of posets was given by R.P. Stanley in [18]. The Stanley-Reisner ideal of this triangulation coincides with a monomial ideal that was shown by T. Hibi [7] to an initial ideal of a toric ideal $J$ associated to the poset $P$. For $P=P_{k, n}$ the ideal $J$ is $J_{k, n}$ and Stanley's triangulation in this case is $\Delta_{k, n}^{N N}$. By the 
correspondence between squarefree initial ideals and regular unimodular triangulations from [19, Ch. 8] it then follows that Stanley's triangulation is also regular. In [13] V. Reiner and V. Welker construct, for every graded poset $P$ of rank $n$, a regular unimodular triangulation $\Delta$ of $\mathcal{O}(P)$ that decomposes as $2^{V} * \widetilde{\Delta}$ for a simplex $2^{V}$ with $n$ vertices and a polytopal sphere $\widetilde{\Delta}$. (A Gorenstein triangulation). They call $\Delta$ the equatorial triangulation of $\mathcal{O}(P)$. The existence of Gorenstein triangulations was later verified by C. A. Athanasiadis [2] for a larger geometrically defined class and then by W. Bruns and T. Römer [3] for the even larger class of all Gorenstein polytopes admitting a regular unimodular triangulation. Gorenstein, here, is equivalent to the $h$-vector of an unimodular triangulation being symmetric, and it was first shown in [7] that an order polytope $\mathcal{O}(P)$ is Gorenstein if and only if $P$ is graded.

In particular, any of [13], [2], [3] shows existence of a regular, unimodular, Gorenstein triangulation of $\mathcal{O}\left(P_{k, n}\right)$. This implies that the multidimensional Narayana numbers are the face numbers of a simplicial polytope, and thus satisfy all conditions of the $g$-theorem. But neither of them guarantees the polytope to be flag. The construction in the present paper does, so the multidimensional Narayana numbers conjecturally also satisfy the Charney-Davis inequalities. Also, none of the previous constructions yields Gorenstein triangulations with the symmetry $\Delta_{k, n}^{N C} \cong \Delta_{n-k, n}^{N C}$ of Proposition 1.6 (ii). It was pointed out to the authors of [13] by C. A. Athanasiadis that the equatorial triangulation of $\mathcal{O}_{2, n}$ is not isomorphic to a dual associahedron and to our best knowledge neither construction from [2] nor from [3] can be used to obtain such a triangulation. Last, it can be checked that the equatorial triangulation is not flag for $P_{k, n}$ and again neither the results from [2] nor from [3] can guarantee such a triangulation. Thus, $\Delta_{k, n}^{N C}$ appears to be more suited for a combinatorial analysis, and more closely related to Graßmannians, than these previous constructions.

Pylyavsky's noncrossing tableaux: In [12], P. Pylyavskyy introduces and studies what he calls noncrossing tableaux, showing that they are as well equinumerous with standard tableaux. The construction therein does not seem to be directly linked to our multidimensional noncrossing complex. For example, Pylyavskyy's noncrossing tableau are not in general monotone along columns, while the tableaux that we biject to facets of $\Delta_{k, n}^{N C}$ in Section 2 are strictly monotone along rows and columns.

\section{The nonnesting and noncrossing decompositions of a tableau}

This section is devoted to the combinatorics of the nonnesting and the noncrossing complex. We study nonnesting and noncrossing decompositions of tableaux, which will then be the main tool in Section 3 to understand the geometry of these complexes.

As defined in the introduction, a tableau of shape $k \times(n-k)$ is a matrix $T \in \mathbb{N}^{k \times(n-k)}$ that is weakly increasing along rows from left to right and along columns from bottom to top. Observe that we still consider rows as labeled from top to bottom (the top row is the first row). This unusual choice makes tableaux of zeros and ones correspond to vectors in $V_{k, n}$ (which we picture as column vectors). For each weakly increasing vector $b=\left(b_{1}, \ldots, b_{k}\right) \in[0, n-k]^{k}$, the tableau having as its $a^{\text {th }}$ row $b_{a}$ zeroes followed by $n-k-b_{a}$ ones corresponds to the increasing vector $\left(b_{1}+1, \ldots, b_{k}+k\right) \in V_{k, n}$. We denote the set of all tableaux of shape $k \times(n-k)$ by $\mathcal{T}_{k, n}$.

In this section we show how to go from a multiset of vectors in $V_{k, n}$ to a tableau, and vice versa. A geometric interpretation of tableaux as integer points in the cone spanned by the order polytope $\mathcal{O}_{k, n}$, in Section 3 , will then lead to a proof that the noncrossing complex triangulates $\mathcal{O}_{k, n}$.

Let $L$ be a multiset of $\ell$ vectors $\left(i_{1 j}, \ldots, i_{k j}\right) \in V_{k, n}(1 \leq j \leq \ell)$. The summing tableau $T=\left(t_{a b}\right)$ 
of the multiset $L$ is the $k \times(n-k)$-matrix $t_{a b}=\#\left\{j \in[\ell]: i_{a j} \leq b+a-1\right\}$. For example, if $L$ is a single vector $I=\left(i_{1} \cdots i_{k}\right)$, then the summing tableau has only zeroes and ones, and $I$ is the vector corresponding to that $0 / 1$-tableau, mentioned above. Observe that a $0 / 1$-tableau is nothing but the characteristic vector of a filter in $P_{k, n}$. For this reason we denote the tableau corresponding to a vector $I$ as $\chi_{I}$. The following lemma can be seen as a motivation for the definition of the summing tableau, and is a direct consequence thereof.

Lemma 2.1 The summing tableau $T$ of a multiset $L$ of vectors in $V_{k, n}$ equals

$$
T=\sum_{I \in L} \chi_{I} \quad \in \mathbb{N}^{P_{k, n}}
$$

In particular, $T$ is a weakly order preserving map from $P_{k, n}$ to the nonnegative integers and thus a tableau.

It is convenient for the following to represent an $\ell$-multiset $L$ of elements from $V_{k, n}$ as the $(k \times \ell)$-table containing the vectors in $L$ as columns, in lexicographic order. For example, let $n=7$ and $k=3$, and consider the multiset given by the $(3 \times 9)$-table $L$ with summing tableau $T$ :

$L=$\begin{tabular}{l|l|l|l|l|l|l|l|l|l|}
1 & 1 & 1 & 1 & 2 & 2 & 2 & 2 & 3 & 5 \\
\hline 2 & 2 & 2 & 3 & 3 & 4 & 4 & 5 & 5 & 6 \\
\hline 3 & 3 & 4 & 5 & 5 & 5 & 5 & 7 & 7 & 7 \\
\hline & 1 & 2 & 3 & 4 & 5 & 6 & 7 & 8 & 9 \\
\hline
\end{tabular}

$T=$\begin{tabular}{|l|l|l|l|}
\hline 3 & 7 & 8 & 8 \\
\hline 2 & 4 & 6 & 8 \\
\hline 1 & 2 & 6 & 6 \\
\hline
\end{tabular}

If $L$ contains the vector $I_{\hat{1}}:=(n-k+1, \ldots, n) \in V_{k, n}$, that vector does not contribute to the summing tableau since $\chi_{I_{\hat{1}}}=0 \in \mathbb{N}^{P_{k, n}}$. We thus set $V_{k, n}^{*}=V_{k, n} \backslash\{(n-k+1, \ldots, n)\}$ for later convenience.

The following result is at the basis of our results about the two simplicial complexes $\Delta_{k, n}^{N N}$ and $\Delta_{k, n}^{N C}$.

Theorem 2.2 Let $T \in \mathcal{T}_{k, n}$. Then there is a unique multiset $\varphi_{N N}(T)$ and a unique multiset $\varphi_{N C}(T)$ of vectors in $V_{k, n}^{*}$ whose summing tableaux are $T$, and such that

- the vectors in $\varphi_{N N}(T)$ are mutually nonnesting, and

- the vectors in $\varphi_{N C}(T)$ are mutually noncrossing.

In order to prove this, we provide two (almost identical) procedures to construct $\varphi_{N N}(T)$ and $\varphi_{N C}(T)$. Let $T=\left(t_{a b}\right) \in \mathcal{T}_{k, n}$ be a tableau, and let $\ell=\max (T)=t_{1, n-k}$ be its maximal entry. We are going to fill a $(k \times \ell)$-table whose columns give $\varphi_{N N}(T)$ and $\varphi_{N N}(T)$, respectively. Since we want each column to be in $V_{k, n}$, we have to fill the $a^{\text {th }}$ row $(a \in\{1, \ldots, k\})$ with numbers in $\{a, \ldots, a+n-k\}$. Moreover, in order to have $T$ as the summing tableau of the multiset of columns, the number $a+b$ must appear in the $a^{\text {th }}$ row exactly $t_{a, b+1}-t_{a, b}$ times, where we use the convention $t_{a, 0}=0$ and $t_{a, n-k+1}=\max (T)$. That is, we do not have a choice of which entries to use in each row, but only on where to put them. Our procedure is to fill the table row by row from top to bottom, inserting the entries $a+1, \ldots, a+n-k$ in increasing order (each of them the prescribed number of times) placing them one after the other into the "next" free box in the $a^{\text {th }}$ row of the table. The only difference between $\varphi_{N N}$ and $\varphi_{N C}$ is how the term "next" is defined. 
- To obtain $\varphi_{N N}$, "next" is simply the next free box from left to right. In the above example, the table gets filled as follows, where we indicated in the top-left corner of each box, the order in which a given entry is inserted into its row of the table

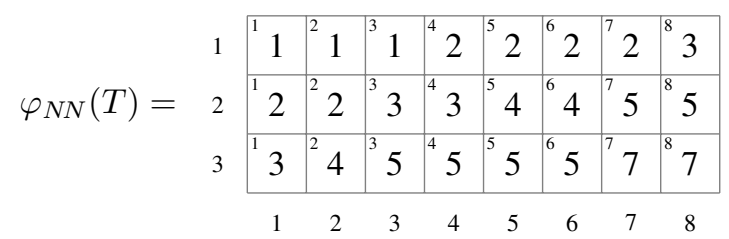

- To obtain $\varphi_{N C}$, "next" is slightly more complicated. For two vectors $v, w \in \mathbb{N}^{k}$ we say that $v$ precedes $w$ in revlex order if the rightmost entry of $w-v$ different from 0 is positive. We chose the revlex-largest vector whose $a^{\text {th }}$ entry has not yet been inserted and for which the property of strictly increasing entries in a column is preserved. In other words, inserting an integer $i$ into row $a$ is done by looking at the first $a-1$ entries $v=\left(v_{1}, \ldots, v_{a-1}\right)$ of all vectors that have not been assigned an $a^{\text {th }}$ entry yet and such that $v_{a-1}<i$. Among those, we assign $i$ to the revlex-largest free box, i.e., to that $v$ for which $v_{a-1}$ is maximal, then $v_{a-2}$ is maximal, and then so on. If this revlexlargest vector is not unique, we fill the box of the left-most of the choices, in order to maintain the table columns in lexicographic order. In the above example, the table now gets filled as

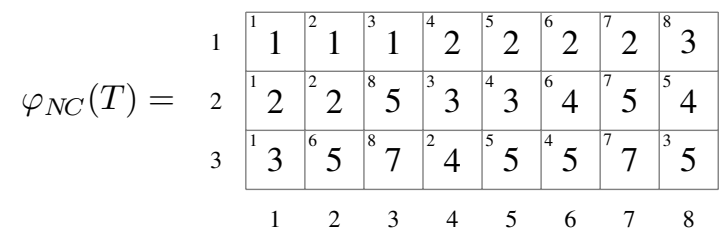

Observe that we could have described the choice of "next" position in the procedure $\varphi_{N N}$ by saying that it means the revlex-smallest vector (in the same sense as above for $\varphi_{N C}$ ) for which the property of strictly increasing entries in a column is preserved. This makes both procedures almost identical, only interchanging revlex-smallest and revlex-largest in the choice of the box to insert the next integer.

Definition 2.3 The multisets $\varphi_{N N}$ and $\varphi_{N C}$ obtained from a tableau $T$ by the above procedures are called the nonnesting decomposition and the noncrossing decomposition of $T$.

Proof of Theorem 2.2; We start with proving that the procedures give what they are supposed to: every two columns of $\varphi_{N N}(T)$ are nonnesting, and every two columns of $\varphi_{N C}(T)$ are noncrossing. To this end, let $I=\left(i_{1}, \ldots, i_{k}\right)$ and $J=\left(j_{1}, \ldots, j_{k}\right)$ be two such columns, and let $a, b$ be two indices such that $i_{\ell}=j_{\ell}$ for all $\ell$ such that $a<\ell<b$. To show that if $I$ and $J$ in $\varphi_{N N}(T)$ (resp. in $\varphi_{N C}(T)$ ) are nonnesting (resp. noncrossing), we have to show that $i_{a}<j_{a}$ implies $i_{b} \leq j_{b}$ (resp. $i_{b} \geq j_{b}$ ). When assigning row $b$ in the table, we see $i_{a}, i_{a+1}, \ldots, i_{b-1}$ in the column containing $I$, and similarly $j_{a}, j_{a+1}, \ldots, j_{b-1}$ in the column containing $J$. In this situation, the column containing $I$ is filled before the column containing $J$ for $\varphi_{N N}$ and after the column $J$ for $\varphi_{N C}$. Thus, $i_{b} \leq j_{b}$ for $\varphi_{N N}$ and $i_{b} \geq j_{b}$ for $\varphi_{N C}$.

To show uniqueness, suppose that we would have not chosen the revlex-smallest (resp. revlex-largest) column at some point in the procedure. The same argument as before then implies that we then would have created two nesting (resp. crossing) columns. 


\section{Geometry of the nonnesting and noncrossing complexes}

Let $Q$ be a polytope with vertex set $V$. A triangulation of $Q$ is a simplicial complex $\Delta$ geometrically realized on $V$ (by which we mean that $V$ is the set of vertices of $\Delta$, and that the vertices of every simplex of $\Delta$ are affinely independent in $Q$ ) that covers $Q$ without overlaps. A triangulation $T$ of a polytope $Q$ is called regular if there is a weight vector $w: V \rightarrow \mathbb{R}$ such that $T$ coincides with the lower envelope of the lifted point configuration

$$
\{(v, w(v)): v \in V\} \subset \mathbb{R}^{|Q|+1} .
$$

See [5] for a recent monograph on these concepts. If the vertices $V$ of the polytope $Q$ are contained in $\mathbb{Z}^{d}$ (or, more generally, in a point lattice) we call a full-dimensional simplex unimodular when it is an affine lattice basis and we call a triangulation unimodular when all its full-dimensional simplices are. All unimodular triangulations of a lattice polytope have the same $f$-vector and, hence, the same $h$-vector. This $h$-vector can be easily computed from the Ehrhart function of $Q$. See, for example, [5, Sect. 9.3.3].

The goal of this section is to show that the noncrossing complex $\Delta_{k, n}^{N C}$ is also a regular, unimodular, flag triangulation of $\mathcal{O}_{k, n}$. (Some details and proofs are omitted due to lack of space, and can be found in the full version of this paper). The method presented recovers the same for the nonnesting complex $\Delta_{k, n}^{N N}$. To do this let us understand a bit more the combinatorics of the facets of $\mathcal{O}_{k, n}$. These are of the following three types:

1. There are two facets corresponding to the unique minimal vector $I_{\hat{0}}:=(1, \ldots, k)$ and the unique maximal vector $I_{\hat{1}}:=(n+1-k, \ldots, n)$. These facets contain each all but one vertices, namely $\chi_{I_{\hat{o}}}=(1, \ldots, 1)$ and $\chi_{I_{\hat{1}}}=(0, \ldots, 0)$. In particular, $\mathcal{O}_{k, n}$ is an iterated pyramid over these two vertices.

2. Each of the $k(n-k-1)$ covering relations $(a, b)-(a, b+1)$ in $P_{k, n}$ produces a facet containing all $\chi_{\left(i_{1}, \ldots, i_{k}\right)}$ with $i_{k+1-a}$ not equal to $k+1-a+b$.

3. Each of the $(k-1)(n-k)$ covering relations $(a, b)-(a+1, b)$ produces a facet containing all $\chi_{\left(i_{1}, \ldots, i_{k}\right)}$ with $i_{k+1-a}<k+1-a+b<i_{k+2-a}$. (That is, vectors in $V_{k, n}$ not containing the entry $k+1-a+b$ and having exactly $a-1$ elements greater than $k+1-a+b$ ).

Denote by $\mathcal{O}_{k, n}^{*}$ the facet containing all vertices of $\mathcal{O}_{k, n}$ except the origin $\chi_{I_{1}}=(0, \ldots, 0)$. Its vertex set is $V_{k, n} \backslash\{(n-k+1 \cdots n)\}$, which is the set $V_{k, n}^{*}$ considered in Section2. Since $\mathcal{O}_{k, n}$ is a pyramid over $\mathcal{O}_{k, n}^{*}$ with apex at the origin, it is natural to consider the cone generated by it, which we denote $\mathcal{C}_{k, n}$. That is,

$$
\mathcal{C}_{k, n}:=\mathbb{R}_{\geq 0} \mathcal{O}_{k, n}=\left\{\lambda \mathbf{v} \in \mathbb{R}^{P_{k, n}}: \lambda \in[0, \infty), \mathbf{v} \in \mathcal{O}_{k, n}\right\} .
$$

Equivalently, $\mathcal{C}_{k, n}$ is the polyhedron obtained from the inequality description of $\mathcal{O}_{k, n}$ by removing the inequality $x_{k, n-k} \leq 1$.

Let us now look at the set $\mathcal{T}_{k, n}$ of all tableaux of shape $k \times(n-k)$, that is, the set of all matrices in $\mathbb{N}^{k \times(n-k)}$ that are weakly increasing along rows from left to right and along columns from bottom to top. It is clear that the inequalities describing weak increase are the same as those defining the facets of the cone $\mathcal{C}_{k, n}$. We hence have the following lemma.

Lemma 3.1 $\mathcal{T}_{k, n}$ is the set of integer points in $\mathcal{C}_{k, n}$. 
Moreover, the summing tableau associated to a list of vectors $I_{1}, \ldots, I_{\ell} \in V_{k, n}^{*}$ is nothing but the sum of the characteristic vectors $\chi_{I_{1}}, \ldots, \chi_{I_{\ell}}$ of the corresponding vertices of $\mathcal{O}_{k, n}$. With this in mind, Theorem 2.2 can be rewritten as follows.

Proposition 3.2 For every integer point $T \in \mathcal{C}_{k, n}$ there is a unique nonnegative integer combination of $V_{k, n}^{*}$ with noncrossing support, and another with nonnesting support, that gives $T$.

Translated into geometric terms, Lemma 3.1 and Proposition 3.2 yield the following corollary.

Corollary $3.3 \Delta_{k, n}^{N N}$ and $\Delta_{k, n}^{N C}$, restricted to $V_{k, n}^{*}$, are flag unimodular triangulations of $\mathcal{O}_{k, n}^{*}$.

Since the origin $\chi_{(n-k+1, \ldots, n)}=(0, \ldots, 0)$ is both noncrossing and nonnesting with every other element of $V_{k, n}, \Delta_{k, n}^{N N}$ and $\Delta_{k, n}^{N C}$ are the pyramids over their restrictions to $V_{k, n}^{*}$. Hence Corollary 3.3 implies that both are unimodular triangulations of $\mathcal{O}_{k, n}$.

Theorem $3.4 \Delta_{k, n}^{N N}$ and $\Delta_{k, n}^{N C}$ are flag unimodular triangulations of $\mathcal{O}_{k, n}$.

To show regularity, consider the following weight function on the set of vertices $V_{k, n}$ of $\mathcal{O}_{k, n}$. For each $I=\left(i_{1}, \ldots, i_{k}\right) \in V_{k, n}$ let

$$
w(I)=w\left(i_{1}, i_{2}, \ldots, i_{k}\right):=\sum_{1 \leq a<b \leq k} \alpha_{b-a} i_{a} i_{b} .
$$

The values $\alpha_{1}, \ldots, \alpha_{n-k+1}$ in the definition are positive and are the same for all $I$. The only condition we require on them is that $\alpha_{i+1} \ll \alpha_{i}$.

Lemma 3.5 Let $(I, J)$ be a pair of noncrossing vertices of $\mathcal{O}_{k, n}$ and let $(X, Y)$ be another pair of (necessarily crossing) vertices with $\chi_{I}+\chi_{J}=\chi_{X}+\chi_{Y}$. Then $w(I)+w(J)<w(X)+w(J)$.

Corollary 3.6 $\Delta_{k, n}^{N C}$ is the regular triangulation of $\mathcal{O}_{k, n}$ produced by the weight vector $w$.

The same ideas can be used to show that the nonnesting complex is the regular triangulation of $\mathcal{O}_{k, n}$ produced by the opposite weight vector $-w$. This means that $\Delta_{k, n}^{N N}$ and $\Delta_{k, n}^{N C}$ are in a sense "opposite" regular triangulations, although this should not be taken too literally. What we claim for this particular $w$ and its opposite $-w$ may not be true for other weight vector $w$ producing the triangulation $\Delta_{k, n}^{N C}$. Anyway, since $\Delta_{k, n}^{N N}$ is the pulling triangulation of $\mathcal{O}_{k, n}$ with respect to any of a family of orderings of the vertices (any ordering compatible with comparability of filters), this raises the question whether $\Delta_{k, n}^{N C}$ is the pushing triangulation for the same orderings. See [5] for more on pushing and pulling triangulations.

Remark 3.7 We saw in Lemma 1.2 that every order polytope has some special faces that are cubes, namely the minimal face containing each pair of vertices. Let $I$ and $J$ be two vertices of $\mathcal{O}_{k, n}$, let $d$ be the number of connected components in $I \triangle J$, and let $\alpha_{I}, \alpha_{J} \in\{0,1\}^{d}$ be the $0 / 1$-vectors identifying $I$ and $J$ as vertices of $\mathcal{F}(I, J)$. Then

- I, J are nonnesting if and only if $\left\{\alpha_{I}, \alpha_{J}\right\}=\{(0, \ldots, 0),(1, \ldots, 1)\}$.

- $I, J$ are noncrossing if and only if $\left\{\alpha_{I}, \alpha_{J}\right\}=\{(0,1,0, \ldots),(1,0,1, \ldots)\}$.

These two triangulations of the cube have been studied in various places before, see [9] Section 2] for a detailed treatment. In particular, the two triangulations $\Delta_{k, n}^{N N}$ and $\Delta_{k, n}^{N C}$ of $\mathcal{O}_{k, n}$ studied in this paper naturally restrict to well studied triangulations of the cubical faces of $\mathcal{O}_{k, n}$.

Acknowledgements: We would like to thank Christian Haase and Raman Sanyal for valuable discussions and the referee for helpful suggestions, and David Speyer for pointing us to the paper mentioned in the "note added in proof" (see Section 1.2). 


\section{References}

[1] C.A. Athanasiadis, On noncrossing and nonnesting partitions for classical reflection groups, Electron. J. Combin. 5 (1998), no. R42.

[2] __ Ehrhart polynomials, simplicial polytopes, magic square and a conjecture of Stanley, J. reine u. angew. Math. 583 (2005), 163-174.

[3] W. Bruns and T. Römer, h-vectors of Gorenstein polytopes, J. Combin. Theory Ser. A 114 (2007), 65-76.

[4] V. I. Danilov, A. V. Karzanov, and G. A. Koshevoy, Separated set-systems and their geometric models, Russian Math. Surveys 65 (2010), no. 4, 659-740.

[5] J. A. De Loera, J. Rambau, and F. Santos, Triangulations, Alg. Comput. Math. 25, Springer, 2010.

[6] K. Górska and K. A. Penson, Multidimensional Catalan and related numbers as Hausdorff moments, Probab. Math. Stat. 33 (2013), no. 2, 265-274.

[7] T. Hibi, Distributive lattices, affine semigroups rings and algebra with straigtening laws, Commutative Algebra and Combinatorics, Adv. Stud. Pure Math. 11, 1987.

[8] M. Joswig and K. Kulas, Tropical and ordinary convexity combined, Adv. Geom. 10 (2010), no. 2, 333-352.

[9] T. Lam and A. Postnikov, Alcoved polytopes I, Discrete Comput. Geom. 38 (2007), 453-478.

[10] B. Leclerc and A. Zelevinsky, Quasi-commuting families of quantum Plücker coordinates, Amer. Math. Soc. Transl. 181 (1998), no. 2, 85-108.

[11] S. Oh, A. Postnikov, and D. E. Speyer, Weak separation and plabic graphs, Preprint, arxiv . org/ abs/1109.4434, 2011.

[12] P. Pylyavskyy, Non-crossing tableaux, Ann. Comb. 13 (2009), no. 3, 323-339.

[13] V. Reiner and V. Welker, On the Charney-Davis and Neggers-Stanley conjectures, J. Combin. Theory Ser. A 109 (2005), no. 2, 247-280.

[14] M. Rubey and C. Stump, Crossings and nestings in set partitions of classical types, Electron. J. Combin. 17 (2010), no. R120.

[15] J.S. Scott, Quasi-commuting families of quantum minors, J. Algebra 290 (2005), no. 1, $204-220$.

[16] _ Grassmannians and cluster algebras, Proc. Lond. Math. Soc. 92 (2006), no. 2, 345-380.

[17] N.J.A. Sloane, On-line encyclopedia of integer sequences, published electronically at oe is . org.

[18] R.P. Stanley, Two poset polytopes, Discrete Comput. Geom. 1 (1986), 9-23.

[19] B. Sturmfels, Gröbner bases and convex polytopes, University Lect. Ser. 8, Amer. Math. Soc., 1996.

[20] R.A. Sulanke, Generalizing Narayana and Schröder numbers to higher dimensions, Electron. J. Comb. 11 (2004), no. R54. 